\title{
Integrative Oncology Care \& Research: Needs to be reinforced
}

Authors:

Raúl H. Morales-Borges, MD (1) (*)

Roberto Román-Juliá, ND, MS (*)

(1) Correspondence Author

(*) Integrative Optimal Health of Puerto Rico

New Alliance Integrative Research

29 Washington Street Suites \# 105 \& $! 07$

San Juan, PR 00907

E-mail addresses:

raul.morales.borges@gmail.com

raul@nairesearch.org

Funding Sources: None

Conflicts of Interest: No conflict of interest to disclose.

Abstract: 115 words

Word Count: 2070

Number of References: 18

Key Words: Integrative Oncology, Care, Research

\begin{abstract}
Healthcare of cancer patients has been changing integrating complementary and alternative medicine (CAM). Since many patients believe in holistic medicine and natural remedies and some studies results demonstrated the efficacy of CAM, it stresses our task to develop innovative research protocols to investigate the outcomes of particular CAM on symptom reduction, quality of life, cancer arrest, or other endpoints. Thus, prospective trials and innovative, evidence-based treatment concepts to include CAM into high-end oncology is what patients demand and what a modern oncology center should offer. We are presenting a review of published studies and experiences with a purpose of motivating investigators to include integrative medicine in the clinical research arena of oncologic patients.
\end{abstract}


Medical Research Archives, Vol. 5, Issue 4, April 2017

Integrative Oncology Care \& Research: Needs to be reinforced

\section{INTRODUCTION}

Cancer affects all age groups and does not discriminate against men, women, or children. Nearly all practitioners-even those not involved directly with oncologyhave seen a person who has or has had cancer in their practice. That's because many cancer patients and survivors are interested in an integrative approach to health. It will help them not only survive but thrive.

We need to start getting involved in clinical trials integrating the traditional medicine with complementary \& alternative medicine (CAM) for cancer patients.

About $50 \%$ of cancer patients use CAM. As per a German study (1), satisfaction is highest ( $>70 \%)$ with patients who use relaxation techniques $(71 \%)$, Vitamin $\mathrm{C}$ (73\%), homeopathy (75\%), yoga (74\%), and Chinese herbs (80\%).

The purpose of Integrative care in oncology patients is focused on increasing the effectiveness of conventional therapies such as surgery, chemotherapy, radiation and reducing the side effects of the combination of the medications. Applying the naturopathic tools in the integrative care of the most oncological patients are intended to extend life and improve the quality of life of the patients. It is a fact proven by scientific studies that blend drugs and surgery with herbal medicine, homeopathy, Traditional Chinese Medicine (TCM), nutrition and mind-body techniques improve the prognosis of patients. That is the reason for major cancer treatment centers in America, Europe, and Asia combine the conventional medicine with naturopathic care guided by a team constituted by a Naturopathic Doctors (ND's), Medical Doctor's (MD's), and an extensive Scientific Experts in health areas.

Integrative care in oncology has improved the response to therapy significantly while lessening complications in oncology hospitals such as Memorial Sloan-Kettering in New York, MD Anderson in Houston, Dana-Farber in Boston, Keith Block's Integrative Cancer Care Institute in Chicago, and all the Cancer Treatment Centers of America hospital and clinics. All these sites share the desire of healing and restoring the balance to allow proper relationships in the physical, emotional, and spiritual dimensions.

\section{STRATEGIES OF NATURO- PATHIC ONCOLOGY}

Some of the primary strategies of naturopathic oncology are:

1. improve the internal biochemistry to support healthy cell division, primarily improving the nutrition

2. stop mutations and support for DNA repair

3. remove the risk factor that promotes cancer: environmental toxins, dietary hazards, emotional stressors

4. inhibit invasion and metastasis

5. support apoptosis

6. management of inflammation and growth factors that speed the progression of diseases

7. destroy malignant tumor using the synergistic forces of non-toxic natural medication with conventional drugs to kill cancer directly

8. inhibit angiogenesis process

9. minimize the side effects and symptoms of the disease and conventional treatments

10. detox from chemo drugs and other harsh medications after treatment. 
Medical Research Archives, Vol. 5, Issue 4, April 2017

Integrative Oncology Care \& Research: Needs to be reinforced

\section{NEEDS FOR CAM STUDIES IN HISPANIC ONCOLOGIC PATIENTS}

The use of CAM among Hispanic adults with the cancer diagnosis has not been well studied and documented yet. David S. Black et al. (2) from Los Angeles, CA, performed a study with 631 patients with colorectal cancer, and they demonstrated that CAM use is common in this population, especially among women, but patient-provider communication about such practices is greatly lacking, presumably because of patient misperceptions surrounding conventional medicine practices.

\section{COST-EFFECTIVENESS OF INTEGRATIVE ONCOLOGY MEDI- CINE}

It is important to let the healthcare professionals know that the integrative oncology care costs less than the traditional care and it will motivate healthcare insurers and institutions to proceed to do integrative care.

According to a study done with breast cancer patients (3), the total costs incurred per year for Bastyr Integrative Oncology Research Center (BIORC) participants ranged from \$1594/year for early-stage breast cancer to more than $\$ 5000 /$ year for stage 3 and 4 breast cancer patients and those who could not be staged at first office call. Costs are highest during the postdiagnosis period when standard oncology treatment is occurring and increases with the stage at BIORC first visit. Of the total amount billed for Integrative Oncology care for one year for 287 breast cancer patients, $21 \%$ was out-of-pocket. Insurance reimbursement paid for $39.1 \%$. The remaining $39.5 \%$ was written off by BIORC. This level of institutional write-off is not unusual. Integrative Oncology (IO) clinics commonly expect at least a 35\% difference between what has been billed to insurers and what insurers pay.

Copyright 2017 KEI Journals. All Rights Reserved
MD Anderson Cancer Center has an Integrative Oncology Program that we visited, and they are doing in-patient and outpatient services integrating physical (medical), mental, and spiritual healthcare professionals. The patients are very satisfied. This model has been implemented in few cancer centers across the United States of America. So, we can continue moving ahead with new IO programs nationally and internationally.

\section{IMPORTANT ISSUES IN CAN- CER PATIENTS}

There are many concepts and matters that the cancer patients have, and we need clinical trials. One important issue is pain management which requires rigorous research $(4,5)$. Cancer patients may be at risk of opioid use disorder, and an integrative review and management are needed for them (5). Another symptom is the cachexia. Understanding and communicating the limitations of naturopathic support therapies when they are initiated late in the course of the cachexia trajectory can reduce unrealistic expectations and decrease burdens on patients and caregivers while educating them on the changes that are occurring. While ongoing and future clinical trials on multimodal treatments for cancer cachexia are much needed and may help us to achieve improvements in outcomes such as quality of life and overall survival for our patients, the synthesis of currently available evidence this article provides serves as a basis and call for routinely employing an integrated approach in an effort to diminish the suffering experienced by those who are at risk for or affected by cancer cachexia (6). Another significant burden in cancer care is the fatigue. One study (7) demonstrated that adding a structured resistive exercise program to the aerobic component will likely lessen fatigue during treatment and lead to overall improvements in quality of life. 
Medical Research Archives, Vol. 5, Issue 4, April 2017

Integrative Oncology Care \& Research: Needs to be reinforced

There are many herbs and natural remedies that we can use, but we still need more research. One example is one study done with intravenous mistletoe (8), but further research should investigate the reported observations.

Integrative Oncology is for the whole person, and Dr. JR Geffen (9) describes six major driving forces behind the wave of transformation presently under way in medicine and health care. He presented a multidimensional approach to cancer care. Gary Deng and Barries Cassileth (10) mentioned above complementary approaches such as mind-body therapies, acupuncture, manipulative and body-based practices, and exercise and physical activity. Combining complementary therapies with mainstream oncology care to address patients' physical, psychologic and spiritual needs constitutes the practice of integrative oncology. I know that many physicians say that integrative medicine integrates a significant amount of pseudoscience and bad science with sciencebased oncology, but it does not need to be this way. It is possible to introduce scientifically supportable elements of CAM into oncology as science- and evidence-based supportive modalities, in essence reversing the rebranding that integrative oncology has been so successful at (11). A German group (12) introduced an integrative oncology expert-based model for breast cancer patients giving them state-of-the-art care.

Another German group (13) evaluated the prevalence and predictors of the use of CAM by cancer patients while being under active treatment with chemo- or radiotherapy or in aftercare. They distributed a standard- ized questionnaire among patients attending a department of radio-oncology, an ambulance for oncology and offices of general practitioners (GPs). Most attributed cancer to stress and trauma (23.7 and $16.4 \%$ ) or genes $(20.8 \%)$. Forty-four percent reported knowing a physician with competence in CAM, and in all settings, most patients named the GP. Fifty-one percent admitted using CAM, $35 \%$ informed the oncologist about using CAM, $56 \%$ told the GP, and $26 \%$ did not notify any physician. Most often used CAM was vitamin D (17\%) and selenium (16\%). Most important goals were to strengthen the immune system $(59 \%)$ and become active (52\%). Most patients were satisfied with the CAM methods they used. With some methods, dissatisfaction was up to $30 \%$. That is important to know that we need more studies to validate it.

\section{EXPERIENCE IN WELL- KNOWN CANCER CENTERS}

In the United States, a center for research into complementary medicine at the National Institutes of Health has a $\$ 68 \mathrm{~m}$ ( $£ 45 \mathrm{~m}$ ) budget and supports a large number of trials and research centers. The United States also has a large number of units for research into complementary medicine that is based at traditional research institutions such as the University of Maryland, Columbia University in New York, Harvard University in Massachusetts, and Memorial Sloan-Kettering Cancer Center in New York (14). Table \#1 demonstrates the cancer centers and few of their clinical research studies and practices related to CAM in the USA. 
Medical Research Archives, Vol. 5, Issue 4, April 2017

Integrative Oncology Care \& Research: Needs to be reinforced

TABLE \# 1: CAM in US Comprehensive Cancer Centers

\begin{tabular}{|c|c|}
\hline Center & Results \\
\hline $\begin{array}{c}\text { Memorial Sloan Kettering Cancer Center } \\
\text { (14) }\end{array}$ & $\begin{array}{l}\text { A phase I trial of an herbal cancer treat- } \\
\text { ment planned at Memorial Sloan-Kettering } \\
\text { has been developed by a team that in- } \\
\text { cludes experts in complementary medicine } \\
\text { research, a statistician who is a specialist } \\
\text { in developing novel designs for phase I } \\
\text { studies, an expert in assessing the quality } \\
\text { of life, and senior oncologists with exten- } \\
\text { sive experience in clinical research. The } \\
\text { researchers have access to a significant } \\
\text { number of patients who are receiving the } \\
\text { highest standard of care. These essential } \\
\text { prerequisites for conducting high-quality } \\
\text { research into complementary medicine } \\
\text { would not have been in place several years } \\
\text { ago. } \\
\text { At the Integrative Medicine Service unit at } \\
\text { MSKCC, practitioners of massage, music } \\
\text { therapy, and acupuncture work in the inpa- } \\
\text { tient wards; patients can be referred by a } \\
\text { doctor, nurse, or social worker. These } \\
\text { treatments are also offered at an outpatient } \\
\text { site along with relaxation, yoga, and t'ai } \\
\text { chi classes. }\end{array}$ \\
\hline H Lee Moffitt Cancer Center (15) & $\begin{array}{l}\text { Use of CAM agents in Glioblastoma } \\
\text { multiforme (GBM) was common, with } \\
77 \% \text { of the cohort reporting CAM usage. } \\
\text { No mortality association was observed } \\
\text { with the use of multivitamins or omega-3 } \\
\text { fatty acids. Patients taking vitamin D as an } \\
\text { individual supplement had reduced mortal- } \\
\text { ity when compared with non-users (age- } \\
\text { adjusted HR }=0.68 \text {; } \mathrm{P}=.02) \text {. However, } \\
\text { the association was diminished after ad- } \\
\text { justment for KPS and ESR (HR }=0.74 \text {; } \mathrm{P} \\
=.09 \text { ). Use of herbal supplements was also } \\
\text { associated with reduced mortality (HR }= \\
0.58 \text {; P =.04). Vitamin E users had an in- } \\
\text { significantly higher mortality when com- } \\
\text { pared with nonusers (HR }=1.54 ; \mathrm{P}=.09 \text { ). }\end{array}$ \\
\hline
\end{tabular}


Medical Research Archives, Vol. 5, Issue 4, April 2017

Integrative Oncology Care \& Research: Needs to be reinforced

\begin{tabular}{|c|l|}
\hline Center & \multicolumn{1}{c|}{ Results } \\
\hline The University of Texas & $\begin{array}{l}\text { One big area of basic research in CAM to } \\
\text { benchmark is on how curcumin directly } \\
\text { targets signaling molecules, as well as the } \\
\text { different forces that bind the curcumin- } \\
\text { protein complex and how this interaction }\end{array}$ \\
affects the biological properties of pro- \\
teins. They also discussed various ana- \\
logues of curcumin designed to bind selec- \\
tive targets with increased affinity. They \\
mentioned inhibition of COX-2, proteo- \\
somes as well as other important enzymes \\
in the cancer cell signaling and metabolim.
\end{tabular}

In the University of Minnesota at Minneapolis (17), two hundred thirty patients were randomized to 1 of 3 groups: therapeutic massage (MT), healing touch (HT), or caring presence $(\mathrm{P})$. All subjects received 4 weekly 45-minute sessions of their assigned intervention (MT, HT, or P) and 4 weekly sessions of a standard care/control. The order of conditions (intervention or control) was randomized. Session 1 of the intervention or control started prior to chemotherapy on the first day of their next scheduled chemotherapy treatment cycle. After the 4 weekly sessions, subjects "crossed over" to the alternate assigned condition for 4 weekly sessions. One subject started on day 15 of treatment and crossed over on day 15 of an identical cycle. The time between weekly visits was a mean of 6.9 to 7.2 days, and the time between crossover periods was a mean of 16.7 days (range, 3-56). About 164 completed all 8 sessions (29\% attrition). Of the 164, 63 received massage, 56 had HT, and 45 were in the presence group. A greater number of subjects assigned to presence dropped prior to the first session because they did not want to be assigned to the presence group. Of the 66 who dropped, 15 were assigned to MT, 21 to HT, and 30 to presence. Half of the subjects dropped because they wanted a different treatment or their schedules changed $(n=33)$, and half were discontinued by the study team because their treatment protocols changed $(\mathrm{n}=30)$ and they no longer met the crossover criteria or they died prior to any treatment $(\mathrm{n}=3)$. All but 3 subjects crossed over on the same day of an identical chemotherapy cycle. This randomized clinical trial design conclusively supported the hypothesis that therapeutic massage and HT were more effective than presence of a caring professional alone or standard care in inducing a relaxed state and reducing short-term pain, mood disturbance, and fatigue in adult patients with cancer undergoing chemotherapy. Participants rated both interventions highly regarding overall helpfulness and satisfaction. There was no clear benefit of one intervention over the other. This was the first published randomized study reporting positive effects of HT in cancer.

\section{ETHICS IN INTEGRATIVE MEDICINE IN CANCER}

During the course of their medical careers, many oncologists and other physicians will encounter patients who are interested in complementary or alternative medical therapies. Though sometimes referred to jointly as CAM, these terms have different mean- 
ings when it comes to their role in a patient's health care. Complementary therapies are used in addition to or integrated with conventional medical treatment, whereas alternative therapies are used instead of conventional medical treatment (18). It can cause some concerns and issues.

It can be difficult for physicians to communicate with patients about alternatives to conventional medical treatment. However, it is important for physicians to set aside their personal opinions and establish open lines of communication with patients who are considering alternative interventions. By discussing alternative interventions, physicians can help patients understand how they compare with standard medical treatments and outcomes. Physicians may also be able to help patients resolve obstacles to receiv- ing standard treatment and distinguish alternatives that are reputable from those that are not. If a patient decides to pursue alternative treatments, the physician should make sure the patient feels free to return for conventional treatment at any time.

\section{CONCLUSIONS}

In conclusion, we need to work with comprehensive IO protocols including IV and oral nutrients and botanicals for clinical research studies looking for response rates, survival rates, toxicity profiles, compliance, and cost-effectiveness as well as integrating psycho-social studies too. We need to eliminate the taboo in this 21 st Century for the benefit of our cancer patients. 
Medical Research Archives, Vol. 5, Issue 4, April 2017

Integrative Oncology Care \& Research: Needs to be reinforced

\section{REFERENCES:}

1. Huebner J, Muenstedt K, Prott FJ, Stoll $\mathrm{C}$, Micke O, Buentzel $\mathrm{J}$ et al. Online Survey of Patients with Breast Cancer on Complementary and Alternative Medicine. Breast Care 2014; 9: 60-63.

2. Black DS, Larn CN, Nguyen NT, Ihenacho U, Figueiredo JC. Complementary and Integrative Health Practices Among Hispanics Diagnosed with Colorectal Cancer: Utilization and Communication with Physicians. The Journal of Alternative and Complementary Medicine 2016; 22(6): 473-479.

3. Standish LJ, Dowd F, Sweet E, Dale L, Andersen MR. Breast Cancer Integrative Oncology Care and Its Costs. Integrative Cancer Therapies 2016; 1-11.

4. Mao JJ, Dusek JA. Integrative Medicine as Standard Care for Pain Management: Th Need for Rigorous Research. Pain Med. 2016 May 26. pii: pnw102.

5. Carmichael AN, Morgan L, Del Fabbro E. Identifying and assessing the risk of opioid abuse in patients with cancer: an integrative review. Subst. Abuse Rehabil. 2016; 7:71-79.

6. Maschke J, Kruk U, Kastrati K, Kleeberg J, Buchholz D, Erickson N, Huebner J. Nutritional care of cancer patients: a survey on patients' needs and medical care in reality. Int. J. Clin. Oncol. 2016 Aug. 2.

7. Cheville AL, Giraldi J, Clark MM, Rummans TA, Pittelkow T, Brown P, Hanson J, Atherton P, et al. Therapeutic exercise during outpatient radiation therapy for advanced cancer: Feasibility and impact on physical well-being. A, J Phys Med Rehabil. 2010; 89(8): 611-619.

8. Kienle GS, Mussler M, Fuchs D, Kiene H. Intravenous Mistletoe Treatment in
Integrative Cancer Care: A qualitative Study Exploring the Procedures, Concepts, and Observations of Expert Doctors. Evid Based Complement Alternat Med 2016;2016:4628287.

9. Geffen JR. Integrative Oncology for the Whole Person: A Multidimensional Approach to Cancer Care. Integrative Cancer Therapies 2010; 9(1): 105-121.

10. Deng G, Cassileth B. Integrative Oncology: An Overview. 2014 ASCO Educational Book; 233-242.

11. Gorski DH. Integrative oncology: really the best of both worlds? Nature Reviews Cancer 2014; 14: 692-700.

12. Dobos GJ, Voiss P, Schwidder I, Choi K, et al. Integrative oncology for breast cancer patients: introduction of an expert-based model. BMC Cancer 2012; 12: 539 .

13. Wortmann J.K., Bremer A., Eich H. et al. Use of complementary and alternative medicine by patients with cancer: a cross-sectional study at different points of cancer care. Med Oncol (2016) 33: 78. doi:10.1007/s12032-016-0790-4.

14. Vickers A. Recent advances: complementary medicine. BMJ. 2000 Sep 16; 321(7262):683-6.

15. Mulpur BH, Nabors LB, Thompson RC, Olson JJ, LaRocca RV, Thompson Z, Egan KM. Complementary therapy and survival in glioblastoma. Neurooncol Pract. 2015 Sep;2(3):122-126. Epub 2015 May 6.

16. Gupta SC, Prasad S, Kim JH, Patchva S, Webb LJ, Priyadarsini IK, Aggarwal BB. Multitargeting by curcumin as revealed by molecular interaction studies. Nat 
Medical Research Archives, Vol. 5, Issue 4, April 2017

Integrative Oncology Care \& Research: Needs to be reinforced

Prod Rep. 2011 November ; 28(12): 1937-1955. doi:10.1039/c1np00051a.

17. Post-White J, Kinney ME, Savik K, Gau JB, Wilcox C, Lerner I.Therapeutic Massage and Healing Touch Improve Symptoms in Cancer. INTEGRATIVE CANCER THERAPIES 2003; 2(4): 332-344.
18. Jacobson GM, Cain JM. Ethical issues related to patient use of complementary and alternative medicine. JOURNAL OF ONCOLOGY PRACTICE 2009; 5(3): 124-126. 